\title{
Exploration of the Pathogenesis of Amyotrophic Lateral Sclerosis from the Perspective of Motor Neuron TDP-43 Protein Expression and ADAR2 Activity
}

\author{
Yu Song ${ }^{a, b}$ Weidong Pan ${ }^{a}$ \\ a Department of Neurology, Shuguang Hospital Affiliated to the Shanghai University of \\ Traditional Chinese Medicine, and ${ }^{\mathrm{b}}$ Shanghai Baoshan District Hospital of Traditional \\ Chinese and Western Medicine, Shanghai, PR China
}

\section{Key Words}

Amyotrophic lateral sclerosis · AMPA receptor · ADAR2 - TDP-43 - Integrative medicine ·

Gene therapy

\section{Abstract}

Amyotrophic lateral sclerosis (ALS) is a common adult-onset nervous system degenerative disease, characterized by the progressive loss of upper and lower motor neurons. TDP-43 pathology in motor neurons is a hallmark of ALS. In addition, the reduced expression of an RNAediting enzyme, adenosine deaminase acting on RNA 2 (ADAR2), increases the expression of GluA2 at an unedited glutamine/arginine $(\mathrm{Q} / \mathrm{R})$ site in the motor neurons of patients with sporadic ALS. The change in the amino acid residue at the Q/R site of GluR2 results in marked alterations in channel properties of AMPA receptors, which increases $\mathrm{Ca}^{2+}$ permeability, and this increase in $\mathrm{Ca}^{2+}$ influx plays a key role in the death of motor neurons. ADAR2 mRNA is a target RNA for TDP-43, and TDP-43 plays a regulatory role in the expression of ADAR2. Recently, researchers have explored the possibility of gene therapy for ALS by upregulating ADAR2 in mouse motor neurons using an adeno-associated virus serotype 9 (AAV9) vector that enables gene delivery to a wide array of central neurons after peripheral administration and observed that the expression of exogenous ADAR2 in the central neurons effectively prevented progressive motor dysfunction. AAV9-ADAR2 rescued the motor neurons from death by normalizing TDP-43 expression. Therefore, this AAV9-mediated ADAR2 gene delivery may enable the development of a gene therapy for ALS.

(C) 2014 S. Karger AG, Basel 


\section{Introduction}

Amyotrophic lateral sclerosis (ALS) is a common adult-onset motor neuron disease, and $>90 \%$ of ALS cases are sporadic. AMPA ( $\alpha$-amino-3-hydroxyl-5-methyl-4-isoxazolepropionate) receptor-mediated excitotoxicity has been proposed to play a role in the death of motor neurons in ALS. Functional AMPA receptors are tetrameric assemblies that are composed of 4 subunits, GluR1, GluR2, GluR3 and GluR4, and are a subtype of ionotropic glutamate receptors. The $\mathrm{Ca}^{2+}$ conductance of AMPA receptors differs markedly depending on whether the receptor has the GluR2 subunit in its subunit assembly. The change in the amino acid residue at the glutamine/arginine (Q/R) site of GluR2 results in marked alterations in the channel properties of AMPA receptors, including $\mathrm{Ca}^{2+}$ permeability, trafficking, subunit assembly and the kinetic aspects of channel gating. These properties of GluR2 are generated by a single nucleotide conversion from adenosine (A) to inosine (I) by posttranscriptional RNA processing called 'RNA editing' (A-to-I editing). Enzymes responsible for the A-to-I conversion have been termed 'adenosine deaminase acting on RNA 2' (ADAR2).

\section{AMPA Receptor-Mediated Neuronal Death}

The failure of GluA2 RNA editing resulting from the downregulation of the RNA-editing enzyme ADAR2 occurs in the majority of ALS cases and causes the death of motor neurons via a $\mathrm{Ca}^{2+}$-permeable AMPA receptor-mediated mechanism (fig. 1) [1, 2].

RNA editing of GluR2 mRNA at the Q/R site was reported in autopsy-obtained spinal motor neurons. Also, it was increased more than unediting in spinal cord motor neurons compared to healthy controls. The editing is catalyzed by ADAR2 enzymes. The ADAR2 expression level was significantly decreased in the spinal ventral gray matter of sporadic ALS patients compared to normal control subjects. It is likely that selective ADAR2 activity in motor neurons induced deficient GluR2 Q/R site editing, which results in neuronal death in sporadic ALS. Thus, it is likely that an increase in the proportion of Q/R site-unedited GluR2containing $\mathrm{Ca}^{2+}$-permeable AMPA receptors and a decrease in edited GluR2 initiates the death of motor neurons in sporadic ALS [3-5].

GluR2 Q/R site editing is specifically catalyzed by ADAR2. Kawahara et al. [3] and Kawak and Kawahara [6] showed that about half of their patients with sporadic ALS motor neurons expressed unedited GluA2 Q/R sites, resulting in reduced ADAR2 activity. Hideyama et al. [7] reported that reduced ADAR2 activity may directly induce motor neuron cell death in ADAR2 knockout mice .

\section{Co-Occurrence of TDP-43 Mislocalization and Abnormal Processing of TDP-43 in Motor Neurons}

TDP-43 is a nuclear protein of 414 amino acids, encoded by the transactive response DNA-binding protein gene on the first autosomal gene. Its molecular weight is $43 \mathrm{kDa}$, and it is thus named 'TDP-43'. TDP-43 contains two RNA recognition sequences. The C-terminal region of TDP-43 is glycine rich and capable of binding directly to several proteins of the heterogeneous nuclear ribonucleoprotein family with well-known splicing inhibitory activity and with the relevant ability of generating mRNA $[8,9]$. Normally, TDP-43 is synthesized in the cytoplasm and then converted into mature and stable peptides. Mostly, it is transported to the nucleus, where it binds to TAR DNA, which plays a role in regulating transcription and alternative splicing. The modification and stabilization of mRNA, degraded by the proteasome 


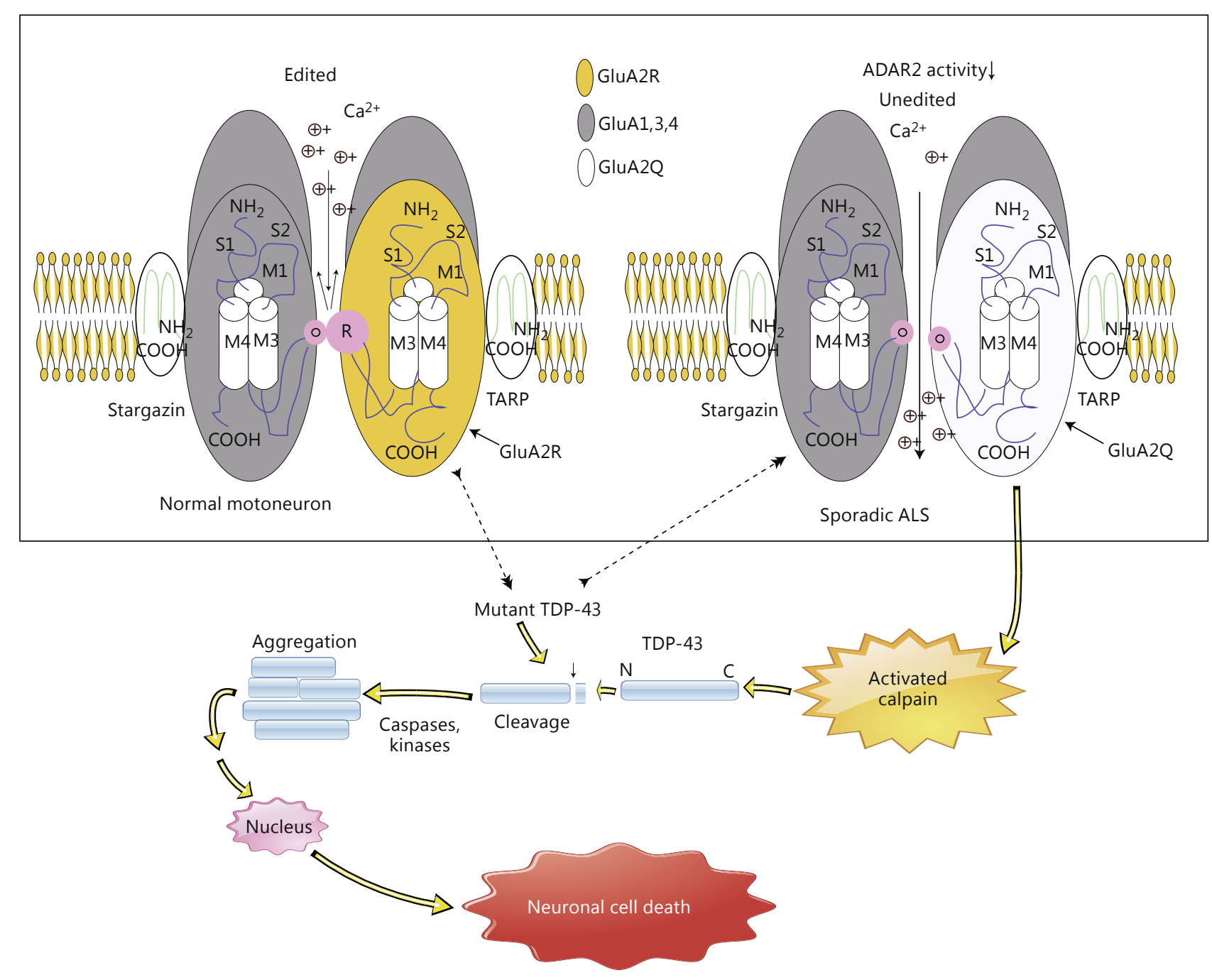

Fig. 1. Diagram illustrating how ADAR2 catalyzes RNA editing at the $Q / R$ site of GluA2. Motor neurons lacking ADAR2 undergo slow death, specifically via a failure of GluA2 RNA editing, which results in the upregulation of $\mathrm{Ca}^{2+}$-permeable AMPA receptors. The increased vulnerability of mutant TDP-43 to calpain-mediated cleavage generates highly aggregation-prone fragments in TARDBP-associated ALS. Higher levels of activated calpain in the spinal motor neurons relative to the cortex and other brain regions may result in the clinical manifestation of ALS. The aggregation-prone nature of calpain-dependent TDP-43 fragments allows them to serve as seeds for cytoplasmic inclusions. Once an aggregate is generated, TDP-43 protein shuttling between the nucleus and the cytoplasm may be sequestered in the inclusions, thereby leading to the death of motor neurons via an as yet undefined cascade.

pathway, result in less cytoplasm with TDP-43 content. The TDP-43 pathogenic mechanism is still unknown; however, two hypotheses have been proposed.

Some scholars believe that the pathogenic mechanism involves the TARDBP gene encoding TDP-43 mutations. Statistics indicate that about 4-6\% of ALS patients possess TARDBP gene mutations; however, the role of mutations in this pathogenesis is unclear, and it is generally believed that the gene mutation affects the normal physiological functions of TDP-43. Some scholars believe that abnormal aggregation and faulty localization are involved 
in the pathogenesis of TDP-43. TDP-43 is a nuclear protein, and abnormal accumulation of the TDP-43 protein could be detected in pathological cytoplasmic inclusions in ALS patients, with the formation of inclusion bodies similar to spherical structures. TDP-43 significantly reduces the expression level of the nucleus. By immunohistochemical detection, a large number of abnormal aggregates in the cytoplasm were shown to be ubiquitin positive, which, normally, cannot be degraded to ubiquitinated aggregates; thus, it is considered that the aggregation is cytotoxic (fig. 1).

The TDP-43 gene is essential for the survival of animals in animal experiments; embryos cannot survive if they are completely missing this gene, and only partial deletion resulted in postnatal death [10]. Normal TDP-43 is positioned within neurons and glial cell nuclei. Its role in the nervous system is still unknown, although current research indicates that it may be a nerve response factor involved in the remodeling of neurons [11, 12]. Studies show that TDP-43 transfers from the nucleus to the cytoplasm, inhibiting transcription and splicing control factors. The lack of TDP-43 will destroy the cellular processes of mRNA splicing in the nucleus. If it is outside the nucleus in the cytoplasm, TDP-43, together with pathological abnormalities, forms inclusion bodies and is cytotoxic. The mechanism by which this protein causes neurological degeneration is still not clear.

Abnormal pathological TDP-43 expression and a localization error in motor neuron cells are specific pathological features of ALS [13], which may be related to the pathogenesis of ALS [14]. Reducing the RNA-editing enzyme (ADAR2) expression in sporadic ALS motor neurons resulted in an increase in the expression of GluA2 unedited at the Q/R sites. The occurrence of these two disease-specific abnormalities in the same motor neurons suggests a molecular link between them. Thus, motor neuron death in ALS patients includes both pathological TDP-43 and reduced ADAR2 activity, and these two pathological changes might be linked to cell death and are therefore related to the pathogenesis of ALS.

Abnormal processing of TDP-43 is not an upstream event of reduced ADAR2 activity. To verify this hypothesis with respect to the pathogenesis of ALS, Yamashita and colleagues $[1,15]$ examined the effects of altered TDP-43 processing on ADAR2 activity in TetHeLaG2m and Neuro2a cells. Human TDP-43 was transfected into both cell lines, and it was found that the overexpression of human TDP-43 in Neuro2a cell lines resulted in a decrease in GluA2 mRNA Q/R site editing, while there was no obvious decline in Q/R site editing in the TetHeLaG2m cell line.

The microarray study by Sephton et al. [16] demonstrated that ADAR2 mRNA is a target RNA for TDP-43. Polymenidou et al. [17] reported that ADAR2 gene expression was reduced in the brain of TDP-43 knockdown mice. These studies suggest a regulatory role for TDP-43 in the expression of ADAR2, which may induce ADAR2 downregulation in ALS motor neurons that lack nuclear TDP-43. However, the extent of ADAR2 downregulation in the TDP-43 knockdown animal brain (reduction by $22 \%$ compared to wild-type mice) [17] was much smaller than that in ALS motor neurons (approximately 70\% compared to control motor neurons) [7,18]. Normal motor neurons express only edited GluA2 (100\% GluA2 Q/R site editing), unless the expression level of ADAR is maintained above half the normal level in mouse motor neurons [19].

Rescue of the ALS Phenotype in a Mouse Model by Intravenous Adeno-Associated Virus Serotype-9-ADAR2 Delivery to Motor Neurons

To explore the possibility of a gene therapy for ALS, Yamashita et al. [15] attempted to broadly deliver ADAR2 cDNA to motor neurons with the aim to enhance the ADAR2 activity to a level sufficient to edit the Q/R site of GluA2 pre-mRNA using an adeno-associated virus serotype 9 (AAV9) vector. AAV9 was transduced to fetal, neonatal and adult animal motor neurons in the spinal cord via intravenous administration $[7,17,20,21]$. Enhancing the 
ADAR2 activity was realized through the delivery of the ADAR2 gene to mouse motor neurons using AAV9 as a vector, together with the SynI promoter, to achieve neuron-specific expression of the ADAR2 gene by injection with AAV9-Flag-human ADAR2 in AR2 mice. AAV9-ADAR2 was successfully delivered to and functioned in motor neurons, and we detected a virtual absence of peripheral expression following intravenous administration in mice. Furthermore, the expression of the delivered ADAR2 prevented the progression of motor dysfunction and neuronal death, with restoration of ADAR2-mediated RNA editing and without inducing any adverse effects in neurons or surrounding tissues in AR2 mice. The death of ADAR2-deficient anterior horn cells (AHCs) is prevented by the expression of Q/R site-edited GluA2 in the absence of ADAR2 in homozygous and heterozygous AR2 mice [17, 22]. The results indicate that AAV9-mediated delivery of human ADAR2 enables the restoration of ADAR2 activity in ADAR2-deficient AHCs to a level that is sufficient to edit the Q/R sites of virtually all the GluA2 pre-mRNAs expressed in AR2 mice. Given that the level of ADAR2 reduction is more modest in the motor neurons of patients with sporadic ALS compared to the AHCs of AR2 mice in which ADAR2 is absent, a therapeutic level of ADAR2 activity would be more easily achieved in ALS patients using this method of gene delivery.

In the AAV9-treated AR2 mice, TDP-43 immunoreactivity was observed to be predominantly nuclear in Flag-positive AHCs, as in normal mouse AHCs, which is in marked contrast to the absence of TDP-43 immunoreactivity or the presence of numerous cytoplasmic TDP-43 immunoreactive aggregates found in ADAR2-lacking AHCs from untreated AR2 mice; TDP-43 pathology in motor neurons of the spinal cord is the pathological hallmark of ALS [19]. Mislocalization of TDP-43 is induced by exaggerated calpain-mediated cleavage of TDP-43 into aggregation-prone fragments, although further activation of calpain cleaves the aggregationprone fragments into smaller soluble fragments [23]. In the case of TDP-43, the degradation process is disturbed. Degradation of the TDP-43 fragment can be transported from the nucleus to the cytoplasm, and then the lysosome of the cytoplasmic fragment degrades them. The proteasome pathway cannot degrade the abnormally cleaved fragments of TDP-43 in the cytoplasm of the easily polymerizable inclusion bodies with ubiquitin and retains them in the cytoplasm. Calpain protease is the degradation protein in the cell enzymes, and its activity is influenced by the calcium concentration in the cytoplasm. ADAR2 deficiency leads to increased permeability of AMPA receptors, and the calcium ion concentration rises in the cytoplasm, leading to abnormal calcium protease activation. Calpain specifically cleaves human and mouse TDP-43, and calpain activation via exaggerated $\mathrm{Ca}^{2+}$ influx through AMPA receptors causes TDP-43 pathology in mouse motor neurons. Calpain serially cleaves TDP-43 into aggregation-prone large fragments and further into smaller fragments that are no longer aggregation prone. The abnormal activation of calpain is a consequence of an increased $\mathrm{Ca}^{2+}$ influx through $\mathrm{Ca}^{2+}$-permeable AMPA receptors containing Q/R site-unedited GluA2 in the ADAR2-deficient AHCs of AR2 mice, and the expression of edited GluA2, even in the absence of ADAR2, rescues TDP-43 mislocalization and AHC loss by normalizing calpain activity.

Yamashita et al. [15] showed that calpain was activated by the downregulation of ADAR2 and that calpain cleaved TDP-43 in the C-terminal region, inducing the generation of aggregation-prone fragments and the mislocalization of TDP-43. TDP-43 mislocalization was reversed by the normalization of calpain activity in AR2res (AR2/GluR-BR/R) mice, in which normal $\mathrm{Ca}^{2+}$-impermeable AMPA receptors were expressed in the absence of ADAR2. Cleavage of TDP-43 was effectively affected by the activity of the endogenous calpain inhibitor CAST in the brains and spinal cords of mice.

Notably, calpain-dependent TDP-43 fragments were present in affected central nervous system tissues of ALS patients, in which calpain activity was increased. These results suggest that an increase in $\mathrm{Ca}^{2+}$ influx through the upregulation of $\mathrm{Ca}^{2+}$-permeable AMPA receptors containing Q/R site-unedited GluA2 activates calpain, and that the exaggerated calpain- 
dependent cleavage of TDP-43 has a crucial role in the process. The findings suggest that calpain-dependent cleavage of TDP-43 is likely an initial event leading to the TDP-43 pathology seen in ALS. However, the underlying mechanisms differ between sporadic ALS and TARDBPassociated ALS: calpain activation through the upregulation of $\mathrm{Ca}^{2+}$-permeable AMPA receptors that contain Q/R site-unedited GluA2 is the theoretical mechanism underlying sporadic ALS, whereas a combination of the increased vulnerability of mutant TDP-43 to calpain with resultant generation of highly aggregation-prone fragments in calpain-rich motor neurons is likely to be the mechanism underlying TARDBP-associated ALS.

Depending on the right AMPA receptor edited at the GluA2 Q/R site, AMPA receptor calcium ion permeability will normally be blocked, and calcium protease will likely show normal activity. Excessive degradation and abnormal aggregation of TDP-43 can be avoided. AAV9-ADAR2-mediated normalization of TDP-43 provides additional evidence that ADAR2 deficiency represents a cause of ALS. Replacement of the deficient ADAR2 protein is a logical approach to therapeutic intervention for ALS that may be effective in the majority of ALS patients.

Researchers have recently developed an ALS mouse model [24], and other results from an Alzheimer's disease mouse model suggest that AAV vector-mediated cDNA delivery to central neurons through the vasculature using neuron-specific promoters as regulators of gene expression is a new therapeutic approach to the treatment of neurodegenerative diseases [25].

\section{Conclusion}

Currently, because GluR2 has a decisive role in AMPA receptor function, it has become an important research field in neurosciences. The GluR2 downregulation mechanism, GluR2 apoptosis in neurons or its toleration still need to be further explored. These questions will help to better prevent and treat a variety of neurological injuries and degenerative diseases.

After years of integrative medicine exploration, therapeutic noninvasive gene treatments delivered to the brain have shown some progress in terms of the carrier and gene combined blood-brain barrier permeability vector carrying the gene into neurons. Gene therapy has been relatively successful in animal models for ALS, but overall, the currently used types of viral and nonviral vectors are limited, and targeting needs to be further improved. With the research and development of novel blood-brain barrier carriers, their applications will become more widespread. Ample experimental evidence will be needed before using this technique clinically in patients.

\section{Acknowledgments}

This study was sponsored and supported by the National Natural Science Foundation of China (81373619) and the Shanghai Pujiang Programme of the Science and Technology Commission of Shanghai Municipality (09PJ1409300).

\section{Disclosure Statement}

The authors declare that there are no conflicts of interest regarding the publication of this article. 
Song and Pan: Exploration of the Pathogenesis of Amyotrophic Lateral Sclerosis from the Perspective of Motor Neuron TDP-43 Protein Expression and ADAR2 Activity

\section{References}

1 Yamashita T, Hideyama T, Teramoto S, Kwak S: The abnormal processing of TDP-43 is not an upstream event of reduced ADAR2 activity in ALS motor neurons. Neurosci Res 2012;73:153-160.

- 2 Kwak S, Hideyama T, Yamashita T, Aizawa H: AMPA receptor-mediated neuronal death in sporadic ALS. Neuropathology 2010;30:182-188.

-3 Kawahara Y, Ito K, Sun H, Aizawa H, Kanazawa I, Kwak S: Glutamate receptors: RNA editing and death of motor neurons. Nature 2004;427:801.

4 Hideyama T, Yamashita T, Suzuki T, Tsuji S, Higuchi M, Seeburg PH, et al: Induced loss of ADAR2 engenders slow death of motor neurons from Q/R site-unedited GluR2. J Neurosci 2010;30:11917-11925.

5 Melcher T, Maas S, Herb A, Sprengel R, Seeburg PH, Higuchi M: A mammalian RNA editing enzyme. Nature 1996;379:460-464.

6 Kwak S, Kawahara Y: Deficient RNA editing of GluR2 and neuronal death in amyotropic lateral sclerosis. J Mol Med 2005;83:110-120.

7 Hideyama T, Yamashita T, Aizawa H, Tsuji S, Kakita A, Takahashi H, et al: Profound downregulation of the RNA editing enzyme ADAR2 in ALS spinal motor neurons. Neurobiol Dis 2012;45:1121-1128.

-8 Buratti E, Brindisi A, Giombi M, Tisminetzky S, Ayala YM, Baralle FE: TDP-43 binds heterogeneous nuclear ribonucleoprotein A/B through its C-terminal tail: an important region for the inhibition of cystic fibrosis transmembrane conductance regulator exon 9 splicing. J Biol Chem 2005;280:37572-37584.

$\checkmark 9$ Hideyama T, Teramoto S, Hachiga K, Yamashita T, Kwak S: Co-occurrence of TDP-43 mislocalization with reduced activity of an RNA editing enzyme, ADAR2, in aged mouse motor neurons. PLoS One 2012;7:e43469.

-10 Tan CF, Eguchi H, Tagawa A, Onodera O, Iwasaki T, Tsujino A, et al: TDP-43 immunoreactivity in neuronal inclusions in familial amyotrophic lateral sclerosis with or without SOD1 gene mutation. Acta Neuropathol 2007;113:535-542.

11 Mackenzie IR, Bigio EH, Ince PG, Geser F, Neumann M, Cairns NJ, et al: Pathological TDP-43 distinguishes sporadic amyotrophic lateral sclerosis from amyotrophic lateral sclerosis with SOD1 mutations. Ann Neurol 2007;61:427-434.

12 Forman MS, Trojanowski JQ, Lee VM: TDP-43: a novel neurodegenerative proteinopathy. Curr Opin Neurobiol 2007;17:548-555.

13 Arai T, Hasegawa M, Akiyama H, Ikeda K, Nonaka T, Mori H, et al: TDP-43 is a component of ubiquitin-positive tau-negative inclusions in frontotemporal lobar degeneration and amyotrophic lateral sclerosis. Biochem Biophys Res Commun 2006;351:602-611.

14 Hasegawa M, Arai T, Nonaka T, Kametani F, Yoshida M, Hashizume Y, et al: Phosphorylated TDP-43 in frontotemporal lobar degeneration and amyotrophic lateral sclerosis. Ann Neurol 2008;64:60-70.

15 Yamashita T, Hideyama T, Hachiga K, Teramoto S, Takano J, Iwata N, et al: A role for calpain-dependent cleavage of TDP-43 in amyotrophic lateral sclerosis pathology. Nat Commun 2012;3:1307.

-16 Sephton CF, Cenik C, Kucukural A, Dammer EB, Cenik B, Han Y, et al: Identification of neuronal RNA targets of TDP-43-containing ribonucleoprotein complexes. J Biol Chem 2011;286:1204-1215.

17 Polymenidou M, Lagier-Tourenne C, Hutt KR, Huelga SC, Moran J, Liang TY, et al: Long pre-mRNA depletion and RNA missplicing contribute to neuronal vulnerability from loss of TDP-43. Nat Neurosci 2011;14:459468.

18 Hideyama T, Kwak S: When does ALS start? ADAR2-GluA2 hypothesis for the etiology of sporadic ALS. Front Mol Neurosci 2011;4:33.

19 Aizawa H, Sawada J, Hideyama T, Yamashita T, Katayama T, Hasebe N, et al: TDP-43 pathology in sporadic ALS occurs in motor neurons lacking the RNA editing enzyme ADAR2. Acta Neuropathol 2010;120:75-84.

20 Dayton RD, Wang DB, Klein RL: The advent of AAV9 expands applications for brain and spinal cord gene delivery. Expert Opin Biol Ther 2012;12:757-766.

-21 Benkhelifa-Ziyyat S, Besse A, Roda M, Duque S, Astord S, Carcenac R, et al: Intramuscular scAAV9-SMN injection mediates widespread gene delivery to the spinal cord and decreases disease severity in SMA mice. Mol Ther 2013;21:282-290.

22 Sawada J, Yamashita T, Aizawa H, Aburakawa Y, Hasebe N, Kwak S: Effects of antidepressants on GluR2 Q/R site-RNA editing in modified HeLa cell line. Neurosci Res 2009;64:251-258.

-23 Foust KD, Nurre E, Montgomery CL, Hernandez A, Chan CM, Kaspar BK: Intravascular AAV9 preferentially targets neonatal neurons and adult astrocytes. Nat Biotechnol 2009;27:59-65.

-24 Cohen TJ, Lee VM, Trojanowski JQ: TDP-43 functions and pathogenic mechanisms implicated in TDP-43 proteinopathies. Trends Mol Med 2011;17:659-667.

25 Iwata N, Sekiguchi M, Hattori Y, Takahashi A, Asai M, Ji B, et al: Global brain delivery of neprilysin gene by intravascular administration of AAV vector in mice. Sci Rep 2013;3:1472. 